\title{
Investigating Developmental Profiles of Children with Autism Spectrum Disorders: Early Indicators and Directions for Intervention
}

\author{
Camilla Teresa Martini Mazetto ${ }^{1,3^{*}}$, Eric Thiébaut ${ }^{2}$, Thais Aquino Vizoná ${ }^{3}$, Maria Clara Nassif ${ }^{3}$, \\ Raissa Ruza dos Santos ${ }^{3}$, Marie-Anna Bernard-Paulais ${ }^{4}$, Jaqueline Wendland ${ }^{1}$, \\ Maria Thereza Costa Coelho de Souza ${ }^{5}$ and Jean-Louis Adrien ${ }^{1}$ \\ ${ }^{1}$ University of Paris, Institute of Psychology, Laboratory of Psychopathology and Health Process, Paris, France \\ ${ }^{2}$ University of Lorraine, Lorraine Laboratory of Psychology and Neurosciences of Behavior Dynamics, Nancy, France \\ ${ }^{3}$ CARI Innovations in Psychology, São Paulo, Brazil \\ ${ }^{4}$ Psychology Office ESPAS-IDDEES, Pont-Ste-Maxence, France \\ ${ }^{5}$ University of São Paulo, Psychology Institute, São Paulo, Brazil \\ Email: contato@camillamazetto.com.br
}

\begin{abstract}
The study describes the relationships between autistic symptomatology severity, developmental heterogeneity, and chronological age for a group of 62 Brazilian children with ASD (59 boys, 3 girls); average chronological age was 42 months and 8 days ( $\mathrm{SD}=17$ months, 15 days). Assessments were carried out with the Social Cognitive Evaluation Battery (SCEB), a French psychological assessment tool currently being validated in Brazil, and the Childhood Autism Rating Scale (CARS). Results indicated a negative correlation between developmental levels and intensity of autistic symptoms; a negative correlation between heterogeneity indices and developmental levels; and a positive correlation between heterogeneity indices and autistic symptomatology intensity. When comparing the group of younger children (less than 33 months) to the older children in the sample, the study demonstrates that early socio-emotional heterogeneity is a developmental marker in young children with ASD, and this finding can lead to personalized intervention programs.
\end{abstract}

Keywords: child development; Autism spectrum disorder; psychological tests, neurodevelopmental disorders, early diagnosis, developmental psychology

\section{Introduction}

The current criteria for the diagnostic classification of Autism Spectrum Disorders (ASD) (American Psychiatric Association [APA], 2013) highlight the wide variety of manifestations of this condition, which affects many neurodevelopmental functions with different intensity (Mehling \& Tassé, 2016). This variability is present not only in the developmental profiles observed from one individual to another, but it is also seen in the significant heterogeneity found in intra-individual development (Lombardo et al., 2019; Uljarević et al., 2018). Intra-individual heterogeneity refers to the different rhythms and developmental characteristics observed in various psychological domains of functioning for one individual, and varies according to chronological age, symptom intensity, and the intellectual impairment of individuals with ASD (Adrien et al., 2016; Bernard et al, 2016).

Describing the developmental profiles of young children with autism and detailing the characteristics of intra-individual heterogeneity can bring new insights into the processes underlying these children's atypical developmental trajectories. The use of standardized psychological assessment tools can provide developmental risk indicators and suggest clinical directions for early developmental intervention (Uljarević et al., 2018). The heterogeneous cognitive and socio-emotional profiles of children with ASD have been described in the literature (Adrien, 1996; Kjellmer at al., 2012) and are observed in different cultures (Bernard-Paulais et al. 2019). The intensity of autistic symptoms is an aggravating factor that impacts both the global development level and intra-individual heterogeneity, as described in many studies on the specific characteristics of psychological development in children with ASD (Chlebowski et al, 2010; Vivanti et al. 2013). Investigating the factors that influence developmental heterogeneity and 
observing longitudinal developmental change can help identify risk factors for abnormal psychological development and directions for individual treatment plans.

The Social Cognitive Evaluation Battery (SCEB) (Adrien, 1996, 2008) is an especially useful tool for assessing the psychological development of children with ASD and their characteristics, the heterogeneity between different cognitive and socio-emotional domains, and developmental trajectories (Boulard et al., 2015; Mazetto, 2015; Schroeder et al., 2015). The SCEB derives from the hypothesis that these children present a significantly heterogeneous development due to disorganized cognitive and socio-emotional development resulting from neurological dysregulation that occurs from the first months of life (Adrien, 1996). Such early differences in basic regulatory processes compromise the development of structures related to cognition, communication, and emotion, undermining the appropriate use of such skills (Adrien et al., 2001, 2007; Blanc et al., 2005).

This study aims to describe the developmental profiles of young Brazilian children with ASD whose developmental ages range from 4 to 24 months using the SCEB, a standardized psychological assessment tool currently being validated in Brazil (Adrien et al., in press). More specifically, this study focuses on the relationship between (a) development levels (cognitive, socio-emotional, and global) and the intensity of autistic symptoms, (b) development levels and heterogeneity indices for the cognitive, socioemotional, and global domains, (c) heterogeneity and intensity of autistic symptoms, and (d) heterogeneity and chronological age.

\section{Methods}

\subsection{Study Design and Participants}

A total of 62 children — 59 boys, 3 girls — with ASD and a developmental level under the age of two years old were included in this study. The developmental level was obtained with the Brunet-Lézine Revised (Souza, 2003), used as part of another international research study protocol (Bernard-Paulais et al., 2019), and with the SCEB (Adrien, 2008). Gender distribution was uneven (59 boys and 3 girls), which reflects the higher frequency of ASD in males. Children's average chronological age was 42 months and 8 days ( $\mathrm{SD}=17$ months, 15 days). The youngest child was 13 months old and the oldest 79 months. A child neurologist and a psychologist specialized in neurodevelopmental disorders diagnosed the participants following the criteria defined by the World Health Organization (2004) and the Diagnostic and Statistical Manual of Mental Disorders (DSM-5) (APA, 2013).

This convenience sample was obtained at a specialized non-profit mental health clinic located in a medically underserved neighborhood in the city of São Paulo (Brazil), dedicated to the diagnosis and treatment of children, adolescents, and adults with ASD. All of the families came from a medium to low socioeconomic background and received subsidized services at the organization. The study was approved by the Ethics Committee for Research in Human Subjects of the Psychology Institute of the University of São Paulo, and the researchers obtained written informed consent from parents or legal guardians.

The children were assessed through clinical observation and the administration of the Childhood Autism Rating Scale (CARS-BR) (Pereira et al., 2008). The researchers used the CARS-BR as a diagnostic instrument and as a measure of the intensity of autistic symptoms. Autism symptom severity was estimated using the CARS-BR, with an average score of 37.26 (SD = 5.59); the cut-off score for an autism diagnosis is 30 points. Children with epilepsy and other neurological disorders, history of perinatal trauma, and known genetic syndromes were excluded from the study.

\subsection{The Social Cognitive Evaluation Battery (SCEB)}

The SCEB (Adrien, 2007) was created from a diagnostic, developmental, and prognostic perspective, in the specific context of the assessment of children with ASD. It assesses the development of children who are over the chronological age of three (older than 3 years old), whose diagnosis of ASD is known, and whose developmental level is comprised between 4 and 24 months. The SCEB is also useful for assessing younger children whose behaviors during the first two years may suggest a neurodevelopmental disorder (Adrien, 2008). This instrument is able to identify subtle developmental progressions and is especially relevant for the longitudinal follow-up of cases diagnosed early (Bernard-Paulais et al., 2019). 
Based on the Piaget (1966) and Fisher (1980) models of child development, the battery comprises 16 domains grouped into two major areas, cognitive development and socio-emotional development. The cognitive area includes 7 domains: self-image, symbolic play, object relation schemata, operational causality, means-ends, spatial relations and object permanence, and the socio-emotional area includes 9 domains: behavior regulation, social interaction, joint attention, expressive language, receptive language, vocal imitation, gestural imitation, affective relations and emotional expression. After the initial rapport, the examiner proposes a series of play activities, following the child's availability, attention, and interests. These activities are intended to highlight cognitive processes (object permanence, causal and spatial relations, symbolic play, among others) and allow the observation of self-regulatory behaviors and interactions thus established by the child with the examiner. The administration lasts approximately 50 minutes.

This assessment tool determines a developmental level in each of the 16 domains, according to a hierarchical list of items for each developmental level: level $1=4-8$ months, level $2=8-12$ months, level $3=12-18$ months, level $4=18-24$ months). Each domain, composed of a minimum of eight items, corresponds to easily observable behaviors that occur during the child's examination or that are described by the parents. Each item is evaluated according to a three-point classification $(0=$ failure; $1=$ emergent behavior or achieved with help; $2=$ stable behavior, achieved without help, complete success). The developmental levels for each assessed domain must represent the most advanced stage attained by the child, i.e., the child has to receive a score of at least 1 (emergent behavior or achieved with help) on that domain if any of the items of that developmental level have scored 1. A developmental level score from 1 to 4 is determined for each of the 16 domains and this provides a developmental profile for each child (Figure 1).

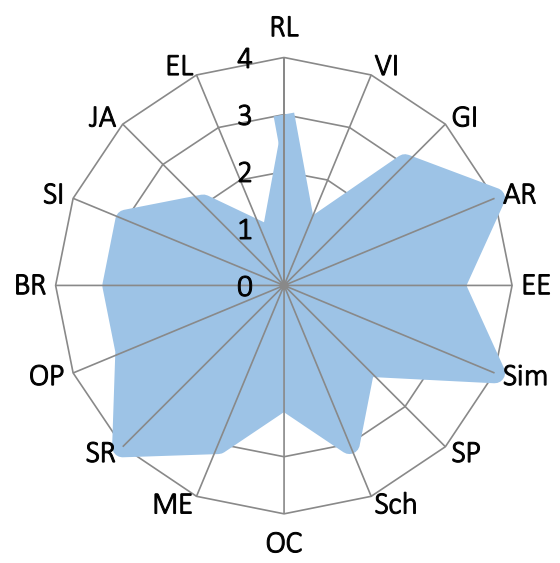

Developmental levels

Figure 1. Cognitive and socio-emotional developmental profile of a boy aged 2 years and 3 months, assessed by the $\mathrm{SCEB}^{\mathrm{a}}$

a Each circle's radius represents one of the 16 domains. The child's developmental level score (0 to 4$)$ is represented by the four concentric circles. Socio-emotional Domains: Behavior Regulation (BR), Social Interaction (SI), Joint Attention (JA), Expressive Language (EL), Receptive Language (RL), Vocal Imitation (VI), Gestural Imitation (GI), Affective Relationships (AR) and Emotional Expression (EE). Cognitive Domains: Self Image (SIm), Symbolic Play (SP), Object relation schemata (Sch), Operational Causality (OC), Means-Ends (ME), Spatial Relations (SR), and Object Permanence (OP).

The SCEB also allows for the calculation of the average global development levels and the average levels of the cognitive and socio-emotional areas, as well as the cognitive (IHC), socio-emotional (IHS), and global (IHG) heterogeneity indices. These indices correspond to the mean difference (in absolute values) between all the level scores (1 to 4 ) of each domain multiplied by 10 . They range from 0 (no heterogeneity) to 16 (maximum heterogeneity). All scores of the SCEB presented appropriate reliability and validity according to the usual psychometric criteria (Adrien, 2007; Thiébaut et al., 2010). 


\subsection{Data Analysis}

This cross-sectional study focused on the correlational analyses of the following variables: (a) between the developmental levels (cognitive, socio-emotional, and global) obtained with the SCEB and the intensity of autistic symptoms (CARS-BR's score), (b) between the developmental levels and the heterogeneity indices (cognitive, socio-emotional and global), (c) between the heterogeneity indices and the intensity of autistic symptoms, and finally (d) between the heterogeneity indices and chronological age.

\section{Results}

\subsection{Correlational Analyses}

Table 1 shows the average scores for the global, cognitive and socio-emotional developmental levels and heterogeneity indices from the SCEB. As expected, in Table 2, it can be noted that there was a significant negative correlation between developmental level - Global (GDL), Cognitive (CDL) and Socio-emotional (SDL) - and autistic symptomatology severity (CARS-BR). This was also the case for heterogeneity indices and global, cognitive and socio-emotional developmental levels, with all correlations being significant at a threshold below .001.

Table 1. Average scores for the global, cognitive, and socio-emotional developmental levels and heterogeneity indices on the $\operatorname{SCEB}(\mathrm{n}=62)$.

\begin{tabular}{lllll}
\hline & Average & SD & Min & Max \\
\hline Global Developmental Level (GDL) & 2,97 & 0,72 & 1,25 & 3,94 \\
Cognitive Developmental Level (CDL) & 3,06 & 0,61 & 1,30 & 4,00 \\
Socio-emotional Developmental Level (SDL) & 2,89 & 0,84 & 1,33 & 4,00 \\
Global Heterogeneity Index (GHI) & 8,49 & 4,17 & 1,25 & 16,91 \\
Cognitive Heterogeneity Index (CHI) & 7,97 & 3,93 & 0,00 & 19,05 \\
Socio-emotional Heterogeneity Index (SHI) & 8,76 & 5,76 & 0,00 & 19,44 \\
\hline
\end{tabular}

Table 2. Correlations between scores on the CARS-BR, developmental levels, and heterogeneity indices ${ }^{\mathrm{a}}$.

\begin{tabular}{lllll}
\hline & CARS-BR & GDL & CDL & SDL \\
\hline CARS-BR & & & & \\
GDL & $-0,60$ & & & \\
CDL & $-0,50$ & & & \\
SDL & $-0,58$ & & & \\
GHI & 0,60 & $-0,82$ & $-0,66$ & $-0,85$ \\
CHI & 0,47 & $-0,71$ & $-0,75$ & $-0,63$ \\
SHI & 0,55 & $-0,73$ & $-0,53$ & $-0,79$ \\
\hline
\end{tabular}

${ }^{a}$ CARS-BR: Children Autism Rating Scale-Brazil; GDL: Global Developmental Level; CDL: Cognitive Developmental Level ; SDL: Socio-emotional Developmental Level; GHI: Global Heterogeneity Index; CHI: Cognitive Heterogeneity Index; SHI: Socio-emotional Heterogeneity Index

The results indicated that lower developmental levels correlate with higher autistic symptomatology severity (higher scores on the CARS-BR). Similarly, the analysis showed a positive correlation between the heterogeneity indices and autistic symptomatology severity, i.e., the greater the global, cognitive, or socio-emotional heterogeneity, the greater the autistic symptomatology severity.

\subsection{Comparative Analysis by Age Group}

We also examined the extent to which a difference in heterogeneity between the cognitive and socioemotional areas varied according to chronological age. Two groups of children were established, with a 
cut-off age of 33 months; this cut-off corresponds to the end of the first psychological development stage in children (Fischer, 1980; Piaget, 1966), and is also the average age above which it is possible to confirm an ASD diagnosis. The youngest group was composed of 24 children (Group 1) and the oldest group of 38 children (Group 2) (Table 3). The correlations between the heterogeneity indices and chronological ages were 0.34 for Group 1 and 0.60 for Group 2. A Fisher's Z-test was used to compare the two independent coefficients and revealed no significant difference $(\mathrm{z}=-1.23$, p. $=0.22)$. A Student's t-test showed no significant difference between cognitive and socio-emotional heterogeneity for Group 2 ( $\mathrm{T}(37)-0.05), \mathrm{p} .=0.96)$, but revealed a significant difference for Group $1(\mathrm{~T}(23)=-2.43$, p. $=0.02)$.

The correlation between autistic symptom severity and cognitive heterogeneity showed no significant difference between the two groups [Group $1=0.26(\mathrm{p} .=0.22)$; group $2=0.53(\mathrm{p} .=0.0006)$; $\mathrm{z}=-1.18$, p. $=0.24]$, but this differed when socio-emotional heterogeneity was considered [Group $1=0.19(\mathrm{p} .=$ 0.38); Group $2=0.67$ (p. $<0.001$ ); $\mathrm{z}=-2,34, \mathrm{p} .=0.02$ ]. The correlation between the Global Developmental Level and the Cognitive Heterogeneity Index was rather similar between the two groups [Group $1=-0.51 ;$ Group $2=-0.40 ; \mathrm{z}=0.31, \mathrm{p} .=0.62]$, which was also the case between the Global Developmental Level and the Socio-emotional Heterogeneity Index (Group $1=-0.83$; Group $2=-0.83$ ).

\subsection{Exploration of Homogeneous Subgroups}

Finally, we sought to classify children according to their similarities on selected variables (chronological age, CARS-BR, Cognitive Developmental Level, and Socio-emotional Developmental Level). A correlation coefficient represents the similarity between two individual profiles. The correlation table between the 62 children in the sample was then subjected to a hierarchical group analysis (the agglomeration method considers the median of the class). The results revealed two groups. The groups were distinguishable by age (group $1=29.75$, group $2=55.6$ ) and overall developmental level (group 1 $=2.69$ and group $2=3.27$ ) but were not different on the CARS-BR scores (group $1=38.27$ and group $2=36.18$ ) and global heterogeneity (group $1=9.33$ and group $2=7.59$ ). The cognitive and socioemotional heterogeneity scores of each group were then compared. Analysis of variance showed a very modest interaction effect $\left(\mathrm{F}(1.60)=3.29, \mathrm{p} .=0.075\right.$, eta $\left.^{2}=0.05\right)$. Cognitive heterogeneity: Group $1=$ 8.01 , Group $2=7.67$; Socio-emotional heterogeneity: Group $1=9.62$, Group $2=8.21$ ).

With a sample size of 62 , a $\alpha$ threshold of .05 (probability of rejecting the null hypothesis when it should not be rejected), and a minimum correlation intensity* (0.47; Table 3), the statistical power of the test (probability of rejecting the null hypothesis when it is actually incorrect) was 0.98. After decomposition into two subgroups, for the minimum size of 24 and a minimum significant correlation of 0.45 , the statistical power of the test was 0.66 . The interpretation of the statistical power indices echoes Levin's (1997)27 proposals that power greater than .51 to detect desired effects would be a reasonable goal, but powers of around .75-.90 would undoubtedly be the best.

Table 3. Descriptive statistics of the cognitive and socio-emotional heterogeneity indices, of two groups of children, categorized by age ${ }^{\mathrm{b}}$.

\begin{tabular}{llll}
\hline & & Average & SD \\
\hline CHI & Group 1 & 7,67 & 3,15 \\
& Group 2 & 8,17 & 4,38 \\
\hline \multirow{2}{*}{ HI } & Group 1 & 9,62 & 3,70 \\
& Group 2 & 8,21 & 6,74 \\
\hline
\end{tabular}

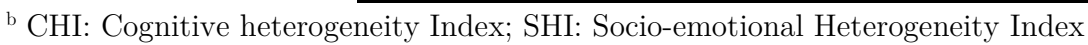

With comparisons of mean and 24 and 38 participants per group, to maintain a statistical power greater than .51, a mean intensity effect must be assumed28 with a d-value for the analysis of .55 .

The heterogeneity of children's developmental profiles seems therefore to fluctuate slightly depending on the product of an interaction between chronological age and overall developmental level. Socioemotional heterogeneity was significantly more important than cognitive heterogeneity in younger children, which means that there were more differences between all the nine socio-emotional domains' 
levels scores than between all the seven cognitive domains' levels scores. To interpret this socioemotional heterogeneity (SHI), an example can be found in Figure 1, representing the socio-emotional developmental profile of a child with ASD (Figure 1). The socio-emotional heterogeneity index $(=11,1$; $\max =16)$ is higher than the cognitive heterogeneity index $(=7,14 ; \max =16)$, which is explained mainly by the very low developmental levels in both expressive language and vocal imitation domains (level $=1$ ) compared to the higher scores in the other seven domains : behavior regulation $($ level $=3)$, joint attention (level $=2$ ), social interaction (level $=3$ ), receptive language (level $=3$ ), gestural imitation (level $=3$ ), emotional expression $($ level $=3$ ) and affective relation $($ level $=4)$.

\section{Discussion}

This study adds new knowledge regarding the early signs of autism and the developmental profiles of ASD children from a developmental perspective. This article also highlights the relevance of performing research with standardized psychological assessment instruments, specially adapted for assessing young children with ASD. Standardized tools such as the SCEB, currently in validation for the Brazilian population (Adrien et al., 2021), are useful for the creation of a common language among childhood mental health professionals, such as pediatricians and child psychologists. They also help establish a more detailed and informative evaluation process and are crucial to help develop evidence-based guidelines for early neurodevelopmental intervention and its longitudinal assessment.

Before discussing the results and concluding, we can indicate some study limitations. Firstly, the sample of children with ASD was small, and since the group of children was from only one Brazilian region, it may not be representative of all Brazilian children with ASD. Moreover, we did not account for parents' socioeconomic status in the analysis. This variable may interfere with children's developmental levels and heterogeneity assessed with the SCEB.

The results indicated a negative correlation between the global, cognitive, and socio-emotional developmental levels and the intensity of autistic symptoms. Thus, the lower the developmental levels, the higher the autistic symptomatology, which corroborates findings by Vivanti et al. (2013) that demonstrated a relationship between the degree of severity of intellectual disabilities and severity of autism. The results also revealed a negative correlation between the heterogeneity indices and developmental levels: that is, the larger the difference between the developmental levels' domains, the lower the mean global cognitive and socio-emotional development levels. Developmental irregularity was therefore associated with low developmental levels. In addition, a positive correlation was observed between the three heterogeneity indices and the intensity of the autistic symptomatology. Thus, the higher the heterogeneity, the greater the intensity of autistic symptoms, which were also related to lower developmental levels.

When comparing the younger children (aged less than 33 months) to the older children in the sample, the socio-emotional heterogeneity was significantly different from the cognitive heterogeneity in the youngest group, but not for children over 33 months old. Adrien et al. (2016) also made this observation, which suggests that socio-emotional heterogeneity is more significant in the early developmental stages of autism's onset. This heterogeneity in the acquisition of socio-emotional skills in early development is noticeable in the optimal socio-emotional skills development period. While it could be considered an early developmental marker in young children with ASD which could be revealed through the SCEB, we noted that this heterogeneity decreases with age.

Furthermore, there was a significant correlation between CARS-BR scores and socio-emotional heterogeneity in older children. When the children were younger (aged between 13 and 33 months), socio-emotional heterogeneity was more significant than cognitive heterogeneity but was not correlated with the intensity of autistic symptomatology. It can be assumed that autistic symptomatology assessed using the CARS-BR in very young children does not score very highly because it is not yet fully expressed in these young children. It would seem that the developmental marker of ASD in young children (under 33 months) is indeed the amplitude of the socio-emotional heterogeneity. This amplitude is no longer a specific marker of ASD after 33 months since it no longer distinguishes itself so clearly from cognitive heterogeneity. Thus, the heterogeneity of children's developmental profiles appears to fluctuate slightly as the product of an interaction of chronological age and overall developmental level. Since in this study, there was no control group of children with ASD who had not received educational 
interventions, it may be that the decrease in cognitive and socio-emotional developmental heterogeneity in these older children with ASD is due to specific neurodevelopmental and psycho-educational interventions having begun at a young age (Nassif, 2017).

\section{Implications}

It seems that the cognitive and socio-emotional development profiles of children with autism spectrum disorder and an overall developmental level of less than two years old are specific in that they reveal a significant socio-emotional heterogeneity in the early stages of development. Detailed and in-depth analyses of these children's developmental profiles must lead to personalized cognitive and socioemotional intervention programs focused on developmentally delayed functions, such as expressive language and imitation, or self-recognition and symbolic play. For children with low developmental levels (1 or 2 on the SCEB) in vocal imitation and language domains and higher developmental levels in social interaction, behavior regulation, and gestural imitation, interventions might focus on these very delayed abilities while considering the potential in social communication and non-verbal imitation. Children with low developmental levels (1 or 2 on the SCEB) in social interaction, behavior regulation, and joint attention might benefit from the Exchange and Development Therapy (Blanc et al., 2013), an early-intervention approach specifically focused on these functions. These individualized programs should be applied in the child's various living environments (Adrien, 2008), such as clinical services and the child's home (Blanc et al., 2013; Nassif, 2017) including the parents (Blanc et al., 2013).

Acknowledgments. The authors would like to thank Pedro H. Neves for helping revise this manuscript and the reference list. The authors would like to thank the Agência USP de Cooperação Acadêmica Nacional e Internacional [USP International Cooperation Office] (AUCANI-USP) for its support.

\section{References}

1. American Psychiatric Association. (2013). Diagnostic and statistical manual of mental disorders (5th ed.). https://doi.org/10.1176/appi.books.9780890425596

2. Adrien, J-L. (1996). L'autisme de l'enfant: Développement psychologique et régulation de l'activité [Childhood autism: Psychological development and activity regulation]. Expansion Scientifique Française; Elsevier.

3. Adrien, J-L. (2007). Manuel de la Batterie d'Évaluation Cognitive et Socio-émotionnelle (BECS) [Manual of the Social- Cognitive Evaluation Battery (SCEB)]. Pearson France-ECPA.

4. Adrien, J-L. (2008). BECS: Pratiques psychologiques et recherches cliniques auprès d'enfants atteints de TED. [SCEB: Psychological practices and clinical research with children with ASD]. De Boeck.

5. Adrien, J-L., Bernard, M-A, Thiébaut, E., Gattegno, M.P, Blanc, Kaye, K., Contejean, Y., Mazetto, C., Nassif, M-C, De Souza, M.T.C.C., Nader-Grosbois, N., Seynhaeve, I., De La Iglesia Gutierriez, M., Olivar Parra, J-S, Aiad, F., Sam, N., Belal, L., Fekih, L., Dionne, C., Rousseau, M., Stefanidou, K., \& Bonnet-Brilhault, F. (2016). Le profil du développement cognitif et socio-émotionnel d'enfants atteints de Troubles du Spectre Autisme (TSA) associé à un léger retard global du développement, âgés de 19 mois à 36 mois diffère-t-il de celui d'enfants avec TSA associé à une déficience intellectuelle sévère et âgés de 7 ans à 14 ans ? Quelles implications pour les pratiques d'évaluation et d'intervention? [Do cognitive and socio-emotional developmental profiles of children with Autism Spectrum Disorders (ASD) with mild developmental delay, aged 19 months to 36 months differ from that of children with ASD and severe intellectual disability and aged 7 to 14 years? What implications for assessment and intervention practices?]. Devenir, 28(3), pp. 255-272.

6. Adrien, J-L., Gattegno, M.P., Kremer, A., \& Abenhaim, N. (2007). L'évaluation psychologique longitudinale dudéveloppement cognitif des enfants avec autisme: Étude de la trajectoire développementale, évaluations et diagnostic. [Longitudinal psychological assessment of cognitive development in children with autism: Study of the developmental trajectory, assessment and diagnosis] ANAE: Approche neuropsychologique des apprentissages chez l'enfant, (91), pp. 41-47.

7. Adrien, J-L., Mazetto, C.T.M., Thiébaut, E., Nassif, M.C., Wendland J. (n.d.). Manual da Bateria de Avaliação Cognitiva e Sócio- Emocional: Validação Brasileira (BECS-BR) [Manual of The Social Cognitive Evaluation Battery (SCEB): Brazilian validation] [in press]. Hogrefe-CETEPP. 
8. Adrien, J-L, Rossignol-Deletang, N., Martineau, J., Couturier, G., \& Barthelemy C. (2001). Regulation of cognitive activity and early communication development in young autistic, mentally retarded, and young normal children. Developmental Psychobiology: The Journal of the International Society for Developmental Psychobiology, 39(2), pp. 124-136.

9. Bernard, M-A, Thiébaut, E., Mazetto, C., Nassif, M-C, De Souza, M.T.C.C., Nader-Grosbois, N., Seynhaeve, I., De La Iglesia Gutierriez, M., Olivar Parra, J-S., Dionne, C., Rousseau, M., Stefanidou, K., Aiad, F., Sam, N., Belal, L., Fekih, L., Blanc, R., Bonnet-Brilhault, F., Gattegno, M.P, Kaye, K., Contejean, Y., \& Adrien, J-L. (2016). L'hétérogénéité du développement cognitif et socio-émotionnel d'enfants atteints d'autisme en lien avec la sévérité des troubles. Revue de neuropsychiatrie de l'enfance et de l'adolescence, (64), pp. 376-382. https://doi.org/10.1016/J.Neurenf.2016.05.002

10. Bernard Paulais, M-A, Mazetto, C., Thiébaut, E., Nassif, M-C, Costa Coehlo De Souza, M.T., Stefani, A.P., Barthelemy, C., Bonnet-Brilhault, F. \& Adrien, J-L (2019). Heterogeneity of cognitive and socio-emotional development of children with autism spectrum disorder and intellectual disability as a comorbidity. Frontiers in Psychiatry, 10, 508. https://doi.org/10.3389/Fpsyt.2019.00508

11. Blanc, R., Adrien, J-L., Roux, S., \& Barthélémy C. (2005). Dysregulation of pretend play and communication development in children with autism. Autism, 9(3), 229-245.

12. Blanc, R., Malvy, J., Dansart, P., Bataille, M., Bonnet-Brilhault, F., \& Barthélémy C. (2013). La Thérapie d'Échange et de Développement : Une rééducation neurofonctionnelle de la communication sociale [Exchange and Development Therapy: A neurofunctional rehabilitation of social communication]. Neuropsychiatrie de l'enfant et de l'adolescent, 61, pp. 288-294.

13. Boulard, A., Morange-Majoux, F., Devouche, E., Gattegno, M.P., Evrard, C., \& Adrien, J-L. (2015). Les dysfonctionnements précoces et les trajectoires développementales d'enfants avec troubles du spectre de l'autisme: une revue des recherches et approches diagnostiques et évaluatives [Early dysfunctions and developmental trajectories of children with Autism Spectrum Disorders: A review of diagnostic and evaluative research and approaches]. Devenir, 27(4), pp. 231-42.

14. Chlebowski, C., Green, J.A., Barton, M.L., \& Fein, D. (2010). Using the childhood autism rating scale to diagnose autism spectrum disorders. Journal of autism and developmental disorders, 40(7), pp. 787-799.

15. Cohen, J. (1988). Statistical power analysis for the behavioral sciences (2nd ed.). Hillsdale, NJ: Erlbaum.

16. Fischer, K.W. (1980). A theory of cognitive development: The control and construction of hierarchies of skills. Psychol Rev., 87(6), 477-531. https://doi.org/10.1037/0033-295X.87.6.477

17. Kjellmer, L., Hedvall, A., Fernell, E., Gillberg, C., \& Norrelgen, F. (2012). Language and communication skills in preschool children with autism spectrum disorders: Contribution of cognition, severity of autism symptoms, and adaptive functioning to the variability. Research in Developmental Disabilities. 33, pp. 172-180.

18. Levin, J. R. (1997). Overcoming Feelings of Powerlessness in "Aging" Researchers: A Primer on Statistical Power in Analysis of Variance Designs. Psychology and Aging, 12(1), pp. 84-106

19. Lombardo, M., Lai, M.C., \& Baron-Cohen, S. (2019). Big data approaches to decomposing heterogeneity across the autism spectrum. Molecular Psychiatry. 24(10), pp. 1435-50.

20. Mazetto, C.T.M. (2015). A criança com autismo: trajetórias desenvolvimentais atípicas à luz da teoria piagetiana da equilibração [The child with autism: atypical developmental trajectories in the light of Piaget's equilibration theory] [Master's thesis]. São Paulo, SP: Universidade de São Paulo.

21. Mehling, M. H., \& Tassé, M.J. (2016). Severity of autism spectrum disorders: Current conceptualization, and transition to DSM-5. Journal of Autism and Developmental Disorders, 46(6), pp. 2000-2016.

22. Nassif, M.C. (Ed.). (2017). Inovações em psicologia clínica: O Programa Abrangente Neurodesenvolvimental [Innovations in clinical psychology: The Comprehensive Neurodevelopmental Program]. Editora Atheneu.

23. Pereira, A., Riesgo, R.S., \& Wagner, M.B. Childhood autism: Translation and validation of the Childhood Autism Rating Scale for use in Brazil. (2008). J Pediatr, 84, pp. 487-94.

24. Piaget J. (1966). La naissance de l'intelligence chez l'enfant [The origins of intelligence in children]. Neuchâtel: Delachaux et Niestlé.

25. Schroeder, C.M., Florence, E., Dubrovskaya, A., Lambs, B., Strittmatter, P., Vecchionacci, V., Bursztejn, C., \& Danion-Grilliat, A. (2015). Le modèle de Denver (Early Start Denver Model). Une approche d'intervention précoce pour les troubles du spectre autistique. [The Early Start Denver Model. An early treatment approach for children with autism spectrum disorder]. Neuropsychiatrie de l'Enfance etdel'Adolescence, 63, pp. 279-287. doi.org/10.1016/j.neurenf.2015.04.001. 
26. Souza, J.M. Avaliação do desenvolvimento neuropsicomotor de crianças de 6 a 24 meses matriculadas em creches municipais de Florianópolis/SC [Assessment of neuropsychomotor development in children from 6 to 24 months enrolled in daycare centers in Florianópolis/SC ]. (2003). [master's thesis]. Florianópolis (SC): UDESC.

27. Thiébaut, E., Adrien, J.L., Blanc, R., \& Barthélémy, C. The Social Cognitive Evaluation Battery for Children with Autism: A New Tool for the Assessment of Cognitive and Social Development in Children with Autism Spectrum Disorders. (2010). Autism Res Treat. ID 875037. doi:10.1155/2010/875037

28. Uljarević, M., Hedley, D., Nevill, R., Evans, D.W., Cai, R.Y., Butter, E., \& Mulick, J.A. (2018). Brief report: Poor self- regulation as a predictor of individual differences in adaptive functioning in young children with autism spectrum disorder. Autism Research,11(8), pp. 1157-1165.

29. Vivanti, G., Barbaro, J., Hudry, K., Dissanayake, C., \& Prior, M. (2013). Intellectual development in autism spectrum disorders: New insights from longitudinal studies. Frontiers in human neuroscience. Jul 5;7, 354.

30. World Health Organization. (2004). ICD-10: International statistical classification of diseases and related health problems (10th revision, 2nd ed.). World Health Organization. 